\title{
Minimization of the Total Traveling Distance and Maximum Distance by Using a Transformed-Based Encoding EDA to Solve the Multiple Traveling Salesmen Problem
}

\author{
S. H. Chen \\ Department of Information Management, Cheng Shiu University, No. 840, Chengcing Road, Niaosong District, \\ Kaohsiung 83347, Taiwan
}

Correspondence should be addressed to S. H. Chen; shchen@csu.edu.tw

Received 15 May 2015; Revised 7 August 2015; Accepted 18 August 2015

Academic Editor: Jason Gu

Copyright (c) 2015 S. H. Chen. This is an open access article distributed under the Creative Commons Attribution License, which permits unrestricted use, distribution, and reproduction in any medium, provided the original work is properly cited.

\begin{abstract}
Estimation of distribution algorithms (EDAs) have been used to solve numerous hard problems. However, their use with in-group optimization problems has not been discussed extensively in the literature. A well-known in-group optimization problem is the multiple traveling salesmen problem (mTSP), which involves simultaneous assignment and sequencing procedures and are shown in different forms. This paper presents a new algorithm, named $\mathrm{EDA}_{\mathrm{MLA}}$, which is based on self-guided genetic algorithm with a minimum loading assignment (MLA) rule. This strategy uses the transformed-based encoding approach instead of direct encoding. The solution space of the proposed method is only $n$ !. We compare the proposed algorithm against the optimal direct encoding technique, the two-part encoding genetic algorithm, and, in experiments on 34 TSP instances drawn from the TSPLIB, find that its solution space is $n !\left(\begin{array}{c}n-1 \\ m-1\end{array}\right)$. The scale of the experiments exceeded that presented in prior studies. The results show that the proposed algorithm was superior to the two-part encoding genetic algorithm in terms of minimizing the total traveling distance. Notably, the proposed algorithm did not cause a longer traveling distance when the number of salesmen was increased from 3 to 10 . The results suggest that EDA researchers should employ the MLA rule instead of direct encoding in their proposed algorithms.
\end{abstract}

\section{Introduction}

Estimation of distribution algorithms (EDAs) use the learning while optimizing principle [1]. Two review articles have suggested that EDAs have emerged as a prominent alternative to evolutionary algorithms $[2,3]$. In contrast to genetic algorithms (GAs), which employ crossover and mutation operators to generate solutions, EDAs explicitly extract global statistical information from the previous search to build a posterior probability model of promising solutions from which new solutions are sampled $[4,5]$. This crucial characteristic distinguishes EDAs from GAs [6,7].

Numerous studies aimed at using EDAs to solve nondeterministic polynomial-time hard (NP-hard) scheduling problems have shown that EDAs are able to perform effectively in terms of the solution quality $[2,8,9]$. Ceberio et al. [2], in particular, extensively tested 13 famous permutationbased EDAs on four combinatorial optimization problems, including the quadratic assignment problem, traveling salesman problem (TSP), permutation flowshop scheduling problems (PFSPs), and linear ordering problem. Their paper provides a good basis for comparison.

Although EDAs are effective in solving various hard problems, EDA studies seldom extensively discuss a problem. To our knowledge, only one EDA, namely, that is proposed by Shim et al. [10], can solve in-group optimization problems such as the multiple traveling salesmen problem (mTSP) and parallel machine scheduling problems (PMSPs) [11]. In-group optimization problems involve assigning and sequencing procedures simultaneously. Take the mTSP, for example: a number of $n$ cities are assigned to $m$ salesmen and these $n$ cities are visited only once by a salesman, where $n>m$. Thus, this appears to be an NP-hard problem.

Because only one EDA could solve in-group optimization problems, there is much room for additional research. Ingroup optimization problems are relevant in industry, such 
as in the application of the mTSP. This research developed a new EDA, named $\mathrm{EDA}_{\mathrm{MLA}}$, dealt with by using a self-guided genetic algorithm (SGGA) [12] with the minimum loading assignment (MLA) rule to solve the mTSP. As opposed to direct encoding, the proposed strategy is called the transformed-based encoding approach. The solution space of the MLA is only $n$ !. We compare the proposed algorithm against the optimal direct encoding technique, the twopart encoding genetic algorithm (TPGA) [13]. Notably, the solution space of the two-part encoding approach is $n$ ! $\left(\begin{array}{c}n-1 \\ m-1\end{array}\right)$. The proposed MLA method, consequently, is superior to the two-part encoding technique, and an improved solution quality is expected when the SGGA works with the MLA method.

This paper is organized as follows: Section 2 primarily reviews the literature on in-group optimization problems, encoding techniques, and EDAs. In Section 3, the core MLA method is presented to dispatch $n$ cities to $m$ salesmen. This assignment rule is further employed by the SGGA in Section 4 . Section 5 reveals the effectiveness of the proposed algorithm, which is compared with the existing famous direct encoding methods, including the one-chromosome and twopart chromosome encoding. Finally, we draw conclusions in Section 6.

\section{Background Information}

The mTSP is a well-known in-group optimization problem. We review mTSP studies and their variants in Section 2.1. To solve in-group optimization problems, numerous encoding techniques could be applied in evolutionary algorithms. Solution representations fall into two classes: direct and indirect encoding methods [11], relevant studies about which are presented in Sections 2.2 and 2.3, respectively. The final section illustrates combinatorial-based EDAs.

2.1. In-Group Optimization Problems. Bektas [11] reviewed the seven types of in-group optimization problems, which we detail in Table 1. Among the variants of in-group optimization problems, the most well-known form is the mTSP because it models daily activities and exists in every enterprise [13]. The problem properties of the mTSP include assignment and sequence optimization procedures. For instance, we must optimize the traveling sequence for the route of each salesman. Both procedures directly lead to the traveling cost and time of the trip after assigning $m$ salesmen to visit $n$ places every day. A detailed definition of the MTSP can be found in [11].

Although the mTSP could be solved using exact algorithms [14-16], large-sized problems are not solved efficiently. To deal with large-size instances, evolutionary algorithms (EAs) are a commonly used approach. The first crucial step of using EAs is selecting the appropriate encodings. Encoding approaches are presented in the next section.

2.2. Direct Encoding Methods. There are five major direct encodings of EAs: one-chromosome [17], two-chromosome
TABLE 1: Application contexts for the in-group optimization problems.

\begin{tabular}{ll}
\hline $\begin{array}{l}\text { Application } \\
\text { context }\end{array}$ & Type of application \\
\hline Routing & mTSP [13, 24, 46-48] \\
\hline Print scheduling & $\begin{array}{l}\text { Print press scheduling [49] } \\
\text { Preprint advertisement scheduling [50] }\end{array}$ \\
\hline Bank crew scheduling [51] \\
Technical crew scheduling [52] \\
Workforce & $\begin{array}{l}\text { Photographer team scheduling [53] } \\
\text { planning }\end{array}$ \\
$\begin{array}{ll}\text { Interview scheduling [54] } \\
\text { Workload balancing [55] }\end{array}$ \\
\hline Security service scheduling [56] \\
$\begin{array}{l}\text { Transportation } \\
\text { planning }\end{array}$ & $\begin{array}{l}\text { Crane scheduling [58] } \\
\text { Local truckload pickup and delivery [59] } \\
\text { Vehicle routing problem [60, 61] }\end{array}$ \\
\hline Mission planning & $\begin{array}{l}\text { Planning of autonomous mobile robots [62-65] } \\
\text { Planning of unmanned air vehicles [66] }\end{array}$ \\
\hline $\begin{array}{l}\text { Production } \\
\text { planning }\end{array}$ & $\begin{array}{l}\text { Hot rolling scheduling [17] } \\
\text { Parallel machine scheduling with setup [29] }\end{array}$ \\
\hline Satellite systems & Designing satellite surveying systems [67] \\
\hline
\end{tabular}

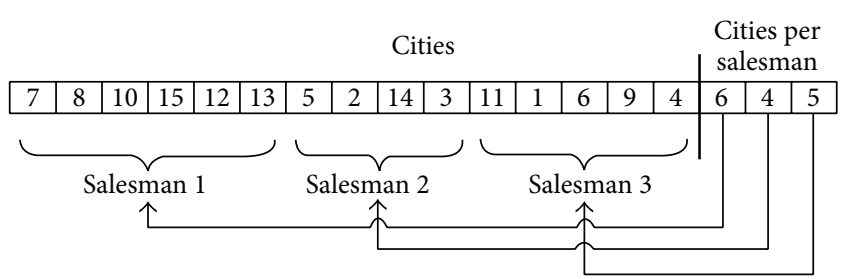

FIGURE 1: A representation of the two-part chromosome encoding for 15 cities and three salesmen.

$[18,19]$, two-part chromosome [13], grouping genetic algorithms (GGAs) [20-22], and matrix representation [23]. Two-part chromosome encoding, which is superior to oneand two-chromosome encoding [13] because of its smaller solution space, is depicted in Figure 1.

We assume this encoding with $n=15$ and $m=3$. There are two distinct parts. The first part of the chromosome represents the permutation of $n$ cities. The second part of the chromosome shows the number of cities assigned to each $m$ salesman so that its chromosome length is $m$. The total sum of $m$ genes is equal to the number of $n$ cities. In [24], they examined an improved combination of crossover and mutation operators for the two-part chromosome encoding method and suggested appropriate genetic operators that could be applied in GAs.

GGAs commonly use an array of jobs for each parallel machine, and the processing order of the jobs assigned to that machine is shown [25]. Kashan et al. [26] extended the GGAs into the grouping version of the particle swarm optimization algorithm. Later, Arnaout et al. [23] proposed a matrix representation of the $N$ jobs on $M$ machines, whose 
size is $M \times N$. Each row indicates the parallel machines and the processing sequence of the jobs on it. When there are no jobs to be processed on a machine, number 0 is inserted into the blank spaces. As a result, it became apparent that GGAs memory usage was inefficient, though Liao et al. [27] found that this approach was better than the other four variants of hybrid ant colony optimization. Thus, $M \times N-N$ spaces are unused if we apply this encoding technique.

In these direct encoding techniques, the optimal approach could be the two-part chromosome technique, according to Carter and Ragsdale [13]. When we have $n$ items and $m$ groups, the solution space of one-chromosome encoding requires $(n+m-1)$ !. Two-chromosome encoding takes $n ! m^{n}$ and the size of the two-part chromosome is $n !\left(\begin{array}{c}n-1 \\ m-1\end{array}\right)$.

2.3. Indirect Encoding Methods. The transformed-based encoding type separates sequencing and assignment decisions because the complex encoding may yield poor results [28]. Its encoding strategy first utilizes permutation encoding and then assigns the items into groups at every stage. Although this approach could be used to solve the PMSP [29], the separated method is also applicable in complex flowshop problems involving numerous parallel machines in the flowshop. Ruiz and Maroto [28] referred to this application as the priority rules for hybrid flowshops. Wang et al. [30] called it the earliest completion factory rule for solving the distributed permutation flowshop scheduling problem. Salhi et al. [31] selected the index of the machine that allows a job that has the shortest completion time for solving complex flowshop scheduling problems.

To achieve optimal efficiency, this study adopts the transformed-based encoding method instead of direct encoding. In addition, several EAs could apply the assignment rule and then solve the in-group optimization problem. To evaluate the performance of the algorithms examined in this study, we select the mTSP for an extensive comparison.

In presenting the latest development in EDAs, it is clear that only a few can solve in-group assignment problems. Thus, this study is relevant to the investigation of in-group assignment problems.

\subsection{Recently Developed Combinatorial-Based EDAs. Unlike} the implicit processing of building blocks in GAs, EDAs explicitly rely on the used probability model. The building blocks are based on selection and crossover operators that do not preserve essential patterns [32]. The probability model is the core factor in affecting the performance of EDAs. The more accurate the probability model is, the more effective the algorithm will be in preventing the disruption of essential building blocks [33]. In general, a distinguishing characteristic of EDAs is their application of the probabilistic model, which is not used by GAs.

Numerous attempts at using EDAs to solve sequencing or combinatorial optimization problems have been made. For example, Chang et al. [34] proposed a hybrid framework to alternate between EDAs and genetic operators for solving the single machine scheduling problem. A position-based univariate probability model was used in the proposed algorithm. The hybrid framework is beneficial, because though EDAs efficiently improve solution quality in the first few runs, the loss of diversity rapidly increases as additional iterations are executed $[7,35,36]$.

Jarboui et al. [37] proposed a hybrid approach, named EDA-VNS, that combined EDAs with the variable neighborhood search (VNS) [38] to solve PFSPs by using the minimization of the total flowtime. Their probabilistic model considered the order of the job queue and the building blocks of the jobs. This was the first attempt to take into account both first- and second-order statistical information. In addition, VNS improved as the EDA was run. Jarboui et al. [37] found that EDA-VNS was effective in small benchmarks; however, for larger problems, VNS was superior to EDAVNS in terms of objective values and computational time. It was unclear why EDA-VNS did not outperform the VNS in large benchmarks. A new EDA in [4] also employed job permutation and similar blocks of jobs to solve lot-streaming flowshop problems. In this EDA, the definitions of job permutation and similar blocks differed from those of [37]; it also introduced a diversity measure to restart evolutionary progress when the population diversity decreased to a certain level.

In contrast to traditional EDAs, an SGGA uses a probabilistic model as the fitness function surrogate [39]. The probabilistic model guides the evolutionary direction in selecting candidate solutions for crossover and mutation operators. An SGGA could solve PFSPs. It could also be integrated with dominance properties to solve single machine scheduling problems [40]. An eSGGA was proposed for problems involving variable interactions [9].

To the best of our knowledge, the first EDA for the mTSP involved applying the one-chromosome representation [10]. Because there are $m-1$ pseudo cities introduced in the chromosome, every chromosome comprises $n+m-1$ genes. As a result, the dimension of their probability model $P_{r}(x)$, by computing the marginal probability of each city, is $N \times N$ where $N$ is $n+m-1$. This might be a drawback of the first EDAs, which were inherited from one-chromosome encoding, even though their performance was superior to three state-of-the-art multiobjective evolutionary algorithms. Consequently, the proposed algorithm $\mathrm{EDA}_{\mathrm{MLA}}$, together with the MLA rule, may be the second EDA to solve the mTSP; it is a promising algorithm that does not use the larger probability model of EDAs.

\section{Minimum Loading Assignment Rule in the mTSP}

Suppose that there is a set of $n$ cities, sequenced $\pi_{1}, \pi_{2}, \ldots, \pi_{n}$ in $\pi$, that could be assigned to $m$ salesmen. These cities are not yet assigned to any salesmen. The sequence $\pi$ could be decoded by using an assignment rule to assign the cities to each salesman. After the assignment rule is executed, we can calculate the fitness function of each chromosome. We propose an MLA rule to perform the assignment work. The following pseudocode in Algorithm 1 illustrates this rule. 


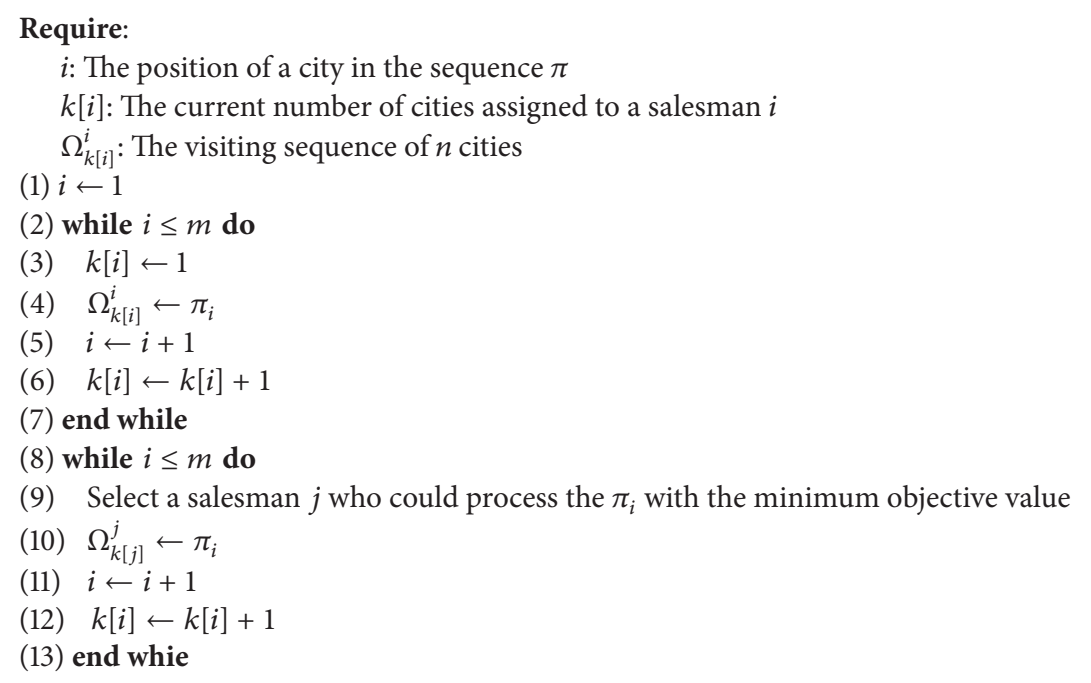

Algorithm 1: Minimum loading assignment rule.

Population: A set of solutions

Generations: The maximum number of generations

$P(t)$ : Probabilistic model

$t$ : Generation index

(1) Initialize Population

(2) $t \leftarrow 0$

(3) Initialize $P(t)$

(5) while $t<$ generations do

(6) EvaluateFitness(Population) with minimum loading assignment rule

(7) Selection/Elitism(Population)

(8) $P(t+1) \leftarrow$ BuildingProbabilityModel(Selected Chromosomes)

(9) Self-Guided Crossover( )

(10) Self-Guided Mutation( )

(11) $t \leftarrow t+1$

(12) end while

Algorithm 2: Main procedures of $\mathrm{EDA}_{\mathrm{MLA}}$.

In the beginning, the first $m$ cities are assigned to $m$ salesmen and the objective values of each salesman are calculated. The objective function of the mTSP would be the total traveling distance or maximum traveling distance among salesman. The MLA rule is then applied iteratively for unassigned cities. The MLA rule assigns the first unassigned city in the sequence $\pi$ to a salesman when it results in the minimum objective value. This assigned city is removed from $\pi$. This process continues until no cities are left in $\pi$.

By using the MLA rule, a city could be assigned to a salesman who has less loading. It also ensures that this assigned city is close to the last city visited by the salesman; a faraway city would not be considered. Through the MLA rule, mTSP can be extended to the PMSP with a setup consideration or the distributed flowshop scheduling problem.

\section{Proposed Algorithm: EDA}

This section explains the procedures of the EDA with the MLA rule. The advantages of the proposed method include preserving the salient genes of the chromosomes and exploring and exploiting optimal searching directions for genetic operators [40, 41]. The major difference between this proposed algorithm and other works is the problem type; other studies have been aimed at solving the sequencing problem, whereas we addressed the grouping and sequencing problems simultaneously. The major procedures of $\mathrm{EDA}_{\mathrm{MLA}}$ are shown in Algorithm 2.

In Step (1), the population is initialized and the sequence of each chromosome is generated randomly. Step (3) builds the probability matrix $P(t)$ with a matrix dimension of $n$ by 
$n$, where $n$ is the problem size. Each $P_{i j}(t)$ is initialized to be $1 / n$, where $n$ is the total number of cities in |Parentset|. This initialization means that all solutions have the same likelihood of being an optimal solution. The reason for such an initialization is that we have no information about the location of promising solutions.

In Step (5), we evaluate the objective value of each solution. In addition, the MLA rule is used here (see Algorithm 1). After all $n$ cities are assigned to $m$ salesmen, the algorithm evaluates the total distance of all salesmen and the maximum distance among the $m$ salesmen. In Step (6), a binary tournament selection is used to select good solutions from the population.

Step (7) forms the probability model $P(t)$ after the selection procedure. The calculation details are outlined in Section 4.1. In Steps (8) and (9), $P(t)$ is employed in the selfguided crossover and mutation operators. The probability model is used as a fitness surrogate, which is shown in Sections 4.2 to 4.4 . We use the two-point partial mapping crossover and swap mutation in the crossover and mutation procedures for solving the MTSP.

The proposed algorithm is explained in the following sections. We first describe the probability model of the EDA and then explain how the probabilistic model guides the crossover and mutation operators.

4.1. Formulation of the Probabilistic Model. The probability model $P(t)$ of the EDA is defined as

$$
P(t)=\left(\begin{array}{ccc}
P_{11}(t) & \cdots & P_{1 n}(t) \\
\vdots & \ddots & \vdots \\
P_{n 1}(t) & \ldots & P_{n n}(t)
\end{array}\right)
$$

where $P_{i j}(t)$ is the probability of city $i$ being in position $j$ in a promising solution. $P(t)$ summarizes the global statistical information about promising solutions obtained from the previous search.

Let $\phi_{i j}$ be the number of solutions in Parentset, in which city $i$ is in position $j$ and |Parentset| is the size of Parentset. $P_{i j}(t+1)$ in Line (7) is updated as follows:

$$
P_{i j}(t+1)=(1-\lambda) P_{i j}(t)+\lambda \frac{\phi_{i j}+1}{\mid \text { Parentset } \mid+n},
$$

where $\phi_{i j} / \mid$ Parentset| is the percentage of solutions in which city $i$ is in position $j$. It represents the knowledge of promising solutions learned from the current generation. We use $\left(\phi_{i j}+\right.$ $1) /(\mid$ Parentset $\mid+n)$, the Laplace correction of $\phi_{i j} / \mid$ Parentset| in (2), to prevent $P_{i j}$ from becoming very small [42-44]. $P_{i j}(t)$ is the historical knowledge of promising solutions. We update $P(t+1)$ in an incremental manner, as suggested by [45]. $\lambda \in(0,1)$ balances the contribution from historical knowledge with that of the knowledge learned from the current generation.
4.2. Probabilistic Model as the Fitness Surrogate. With the probabilistic model $P(t+1)$, we define the following function to predict the quality of solution $X$ :

$$
Q_{t+1}(X)=\prod_{k=1}^{n} P_{k[k]}(t+1),
$$

where $[k]$ is the position of city $k$ in $X$. The following should be noted regarding this function:

(i) $P_{k[k]}(t+1)$ is the probability that city $k$ in position $[k]$ is a promising solution. Therefore, $Q_{t+1}(X)$ can measure how promising $X$ is.

(ii) In general, $Q_{t+1}(X)$ is not an exact probability measure of the set of all the solutions of $X$ because

$$
\sum_{X} Q_{t+1}(X) \neq 1
$$

$Q_{t+1}(X)$ is only an estimation value of the probability that $X$ is promising. This estimation is more effective and much easier to compute compared with other probabilistic models in the literature. Thus, this method is effective and reduces computational time.

$Q_{t+1}(X)$ is applied to select good candidate solutions during the crossover and mutation operation. In the following subsection, we drop $t+1$ in $P$ and $Q$ for simplicity.

4.3. Crossover Operator with Probabilistic Model. With the surrogate function in (3), we preevaluate the solution quality of the new solutions generated by the crossover and mutation operators. In the normal two-point crossover procedure, two random cut-points, $K$ and $L$, are set in the beginning, where $K$ is less than $L$. Then, a parent solution $X$ mates with the other parent solution to yield a new offspring. However, a difference exists in the proposed algorithm.

Because of the difference, we let a parent solution $X$ mate with a set of randomly selected solutions $Y$. The size of $Y$ ranges from 2 to TC, where TC is the number of tournaments. These crossover steps produce a set of offspring $Z$. The quality difference between offspring $Z_{i}$ and parent solution $X$ is denoted as $\Delta_{i} . \Delta_{i}$ is given as follows:

$$
\begin{aligned}
\Delta_{1} & =Q(Z)-Q(X) \\
& =\left[\prod_{K \leq k \leq L} P_{y_{i} i}-\prod_{K \leq k \leq L} P_{x_{i} i}\right] \prod_{1 \leq i<K} P_{x_{i} i} \prod_{L<i \leq n} P_{x_{i} i} .
\end{aligned}
$$

The larger $\Delta_{i}$ is, the more likely that $Z_{i}$ is superior to other offspring when a set of parent solutions $Y$ mate with a solution $X$. Hence, $Z_{i}$ is added to the offspring population. We repeat the crossover steps to generate offspring until the offspring population is full. Both the concepts of self-guided crossover and self-guided mutation employ the same idea. The mutation procedure is shown in the next section.

4.4. Mutation Operator with Probabilistic Model. Suppose that two cities $i$ and $j$ are randomly selected and they are located in position $a$ and position $b$, respectively. $p_{i a}$ and 
$p_{j b}$ denote city $i$ in position $a$ and city $j$ in position $b$. After these two cities are swapped, the new probabilities of the two cities become $p_{i b}$ and $p_{j a}$. The probability difference $\Delta_{i j}$ is calculated as (6), which is a partial evaluation of the probability difference because the probability sum of the other cities remains the same:

$$
\begin{aligned}
\Delta_{i j} & =Q\left(X^{\prime}\right)-Q(X) \\
& \approx \prod_{p \notin(a \text { or } b), g=[p]}^{n} P_{t+1}\left(X_{g p}\right)\left[\left(p_{i b} p_{j a}\right)-\left(p_{i a} p_{j b}\right)\right] .
\end{aligned}
$$

Now because the part of $\prod_{p \notin(a \text { or } b), g=[p]}^{n} P_{t+1}\left(X_{g p}\right)$ is always $\geq 0$, it can be subtracted, and (6) is simplified as follows:

$$
\begin{aligned}
\Delta_{i j} & =\left(p_{i b} p_{j a}\right)-\left(p_{i a} p_{j b}\right), \\
\Delta_{i j} & =\left(p_{i b}+p_{j a}\right)-\left(p_{i a}+p_{j b}\right) .
\end{aligned}
$$

If $\Delta_{i j}$ is positive, it implies that one gene or both genes might move to a promising area. On the other hand, when $\Delta_{i j}$ is negative, the implication is that at least one gene moves to an inferior position.

On the basis of the probabilistic differences, it is natural to consider different choices of swapping points during the mutation procedure. A parameter TM is introduced for the self-guided mutation operator, which denotes the number of tournaments in comparing the probability differences among the TM choices in swap mutation. Basically, TM $\geq 2$ while $\mathrm{TM}=1$ implies that the mutation operator mutates the genes directly without comparing the probability differences among the different TM choices.

When $\mathrm{TM}=2$, suppose the other alternative is that two cities $m$ and $n$ are located in position $c$ and position $d$, respectively. The probability difference of exchanging cities $m$ and $n$ is

$$
\Delta_{m n}=\left(p_{m d}+p_{n c}\right)-\left(p_{m c}+p_{n d}\right) .
$$

After $\Delta_{i j}$ and $\Delta_{m n}$ are obtained, the difference between the two alternatives is as follows:

$$
\Delta=\Delta_{i j}-\Delta_{m n} .
$$

If $\Delta<0$, the contribution of swapping cities $m$ and $n$ is better, so we swap cities $m$ and $n$. Otherwise, cities $i$ and $j$ are swapped. Consequently, the option of a larger probability difference is selected and the corresponding two cities are swapped. By observing the probability difference $\Delta$, the self-guided mutation operator exploits the solution space to enhance the solution quality and prevent destroying some dominant genes in a chromosome. Moreover, the main procedure of the self-guided mutation is (9), where the timecomplexity is only a constant after the probabilistic model is employed. This approach proves to work efficiently.

To conclude, the $\mathrm{EDA}_{\mathrm{MLA}}$ is obviously different from the previous EDAs. Firstly, the algorithm utilizes the transformed-based encoding instead of using the direct encoding used by Shim et al. [10]. Secondly, the proposed algorithm explicitly embeds the probabilistic model in the crossover and mutation operators to explore and exploit the solution space. Most important of all, the algorithm works more efficiently than previous EDAs [10] in solving the mTSP because the time-complexity is $O(n)$ whereas the previous EDAs need $O\left(n^{2}\right)$ time. They are the major differences to other existing EDAs.

\section{Experimental Results}

We conducted extensive computational experiments to evaluate the performance of $\mathrm{EDA}_{\mathrm{MLA}}$ in solving the mTSP. There were 34 instances selected from the well-known traveling salesman problem library, TSPLIB, and the size of these instances ranged from 48 to 400 . This paper assumed that the first city of each instance was the home depot. The number of salesmen used was 3, 5, 10, and 20. Hence, there were 136 instances in the experiments. Across all the experiments, we replicated each instance 30 times.

The proposed algorithm was compared with the benchmark encoding algorithm and a classic encoding, which are the TPGA [13] and one-chromosome genetic algorithm [17], respectively. We employed the genetic operators and parameter settings of the TPGA suggested by S.-H. Chen and M.-C. Chen [24], because they used the design-ofexperiments (DOE) to select significant parameters; the genetic operators are the two-point partial mapping crossover operator and swap mutation operator. This ensures a fair comparison between the proposed algorithm and benchmark encoding algorithm. One-chromosome GA utilizes the same operators of TPGA and also employs the DOE to tune the parameters as well. The crossover and mutation rate of the one-chromosome GA are 0.5 and 0.1 , respectively. Finally, a standard genetic algorithm also applies the MLA rule, which is named $G_{M L A}$. $G_{M L A}$ could show whether the performance is enhanced by the assignment rule proposed by this research.

We implemented the algorithms in Java 2 on an Amazon EC2 with a Windows 2012 server (32-core CPU). The stopping criterion is the number of examined solutions which is up to 100,000 . The objective functions include minimizing the total traveling distance and maximizing the traveling distance, which are detailed in Sections 5.1 and 5.2, respectively.

5.1. Total Traveling Distance Results. This objective evaluates the total distance traveled by the $m$ salesmen. It reflects the total cost of the assignment. Figure 2 shows the main effects plot of the method comparison and the differences in the number of salesmen assigned. This figure clearly illustrates that the $\mathrm{EDA}_{\mathrm{MLA}}$ and $\mathrm{GA}_{\mathrm{MLA}}$ are superior to the onechromosome GA and TPGA. This indicates that the MLA rule, that is, the transformed-based method, could be a more promising approach than the direct encoding methods. The total distance increased greatly with the number of salesmen, particularly when 20 salesmen could be assigned. This implies that the request of too many salesmen would be inefficient from a managerial perspective.

Figure 3 depicts the interaction plot between the factor method and number of salesmen. Notably, the EDA $\mathrm{ELA}_{\mathrm{A}}$ 

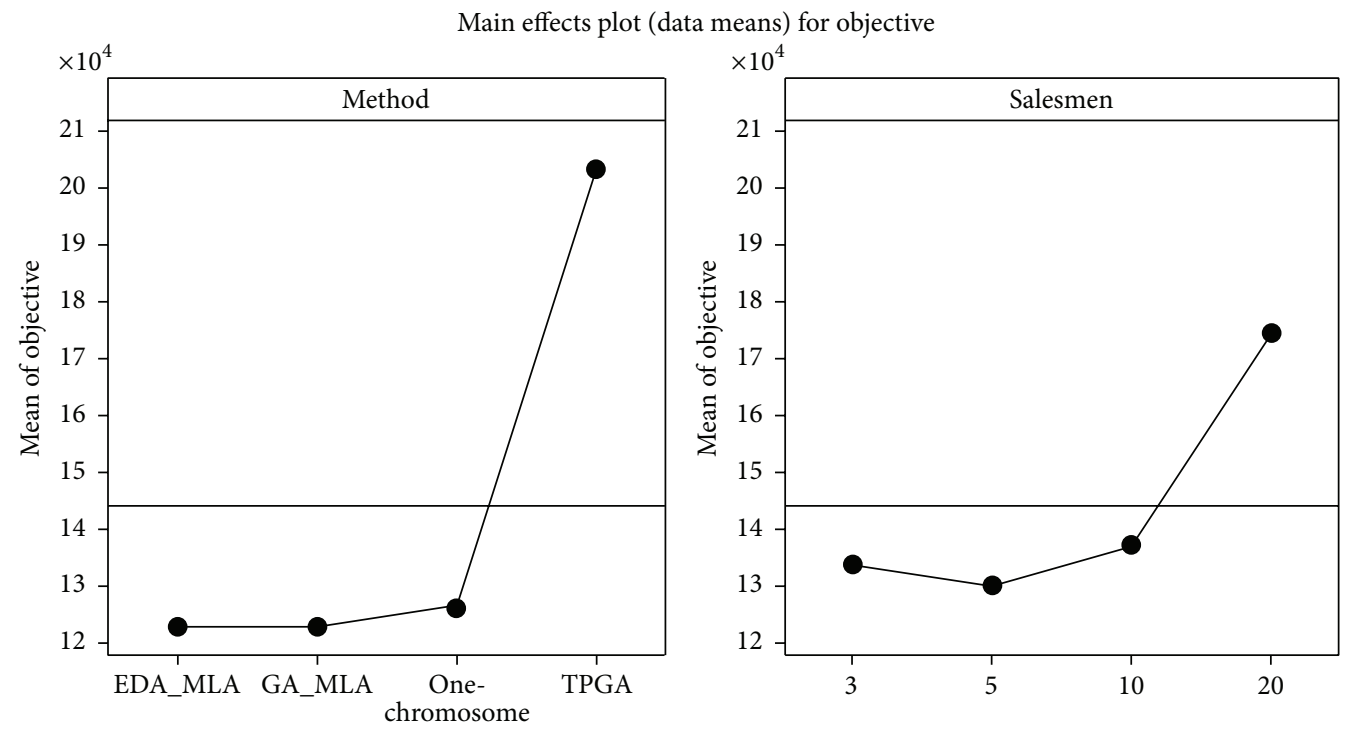

FIGURE 2: Main effects plot on the total travelling distance of the compared algorithms.

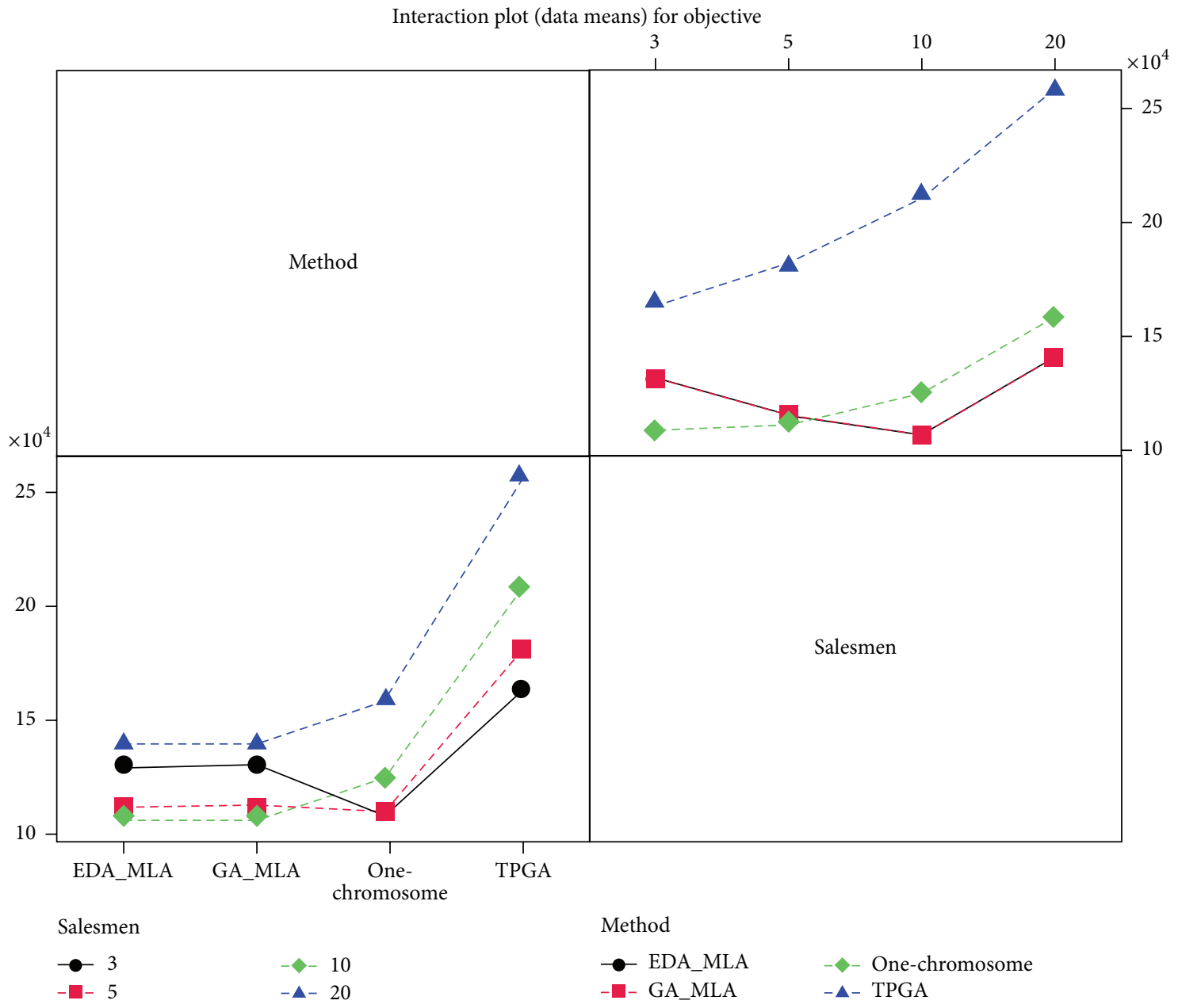

FIGURE 3: Intreaction plot on the total travelling distance. 

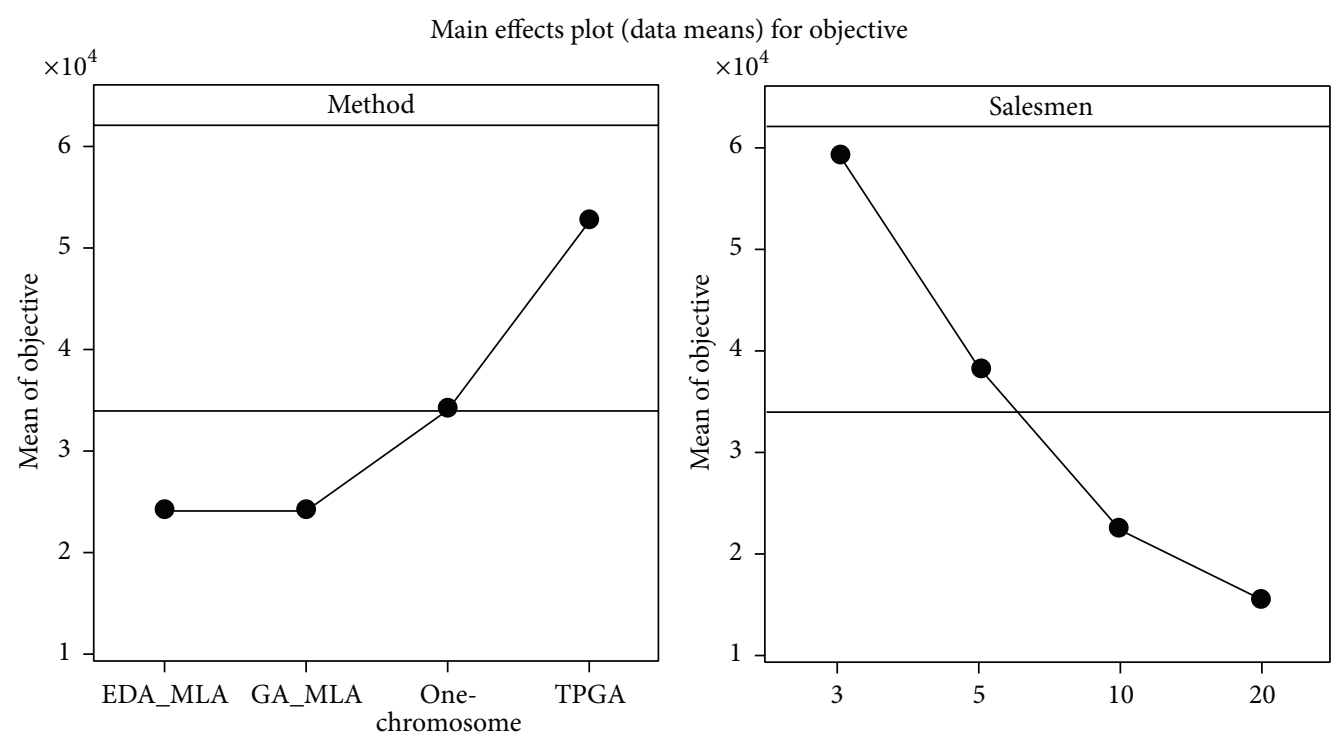

FIGURE 4: Main effects plot of the maximum traveling distance for the compared algorithms.

and $\mathrm{GA}_{\mathrm{MLA}}$ did not yield a longer total traveling distance when the number of salesmen increased from two to 10 . However, the TPGA suffered when the number of salesmen was increased. Thus, this figure reveals the effectiveness of the transform-based rule compared with the direct encoding method. According to this interaction plot, if a manager wants to determine how many salesmen are required, the lowest total traveling distance would be 5 .

Table 2 presents the results of the total traveling distance for the four algorithms. This table shows the minimum, mean, maximum, and the standard deviation (StDev). Among these 34 instances, $\mathrm{EDA}_{\mathrm{MLA}}$ is better than one-chromosome GA, TPGA, and $\mathrm{GA}_{\mathrm{MLA}}$ out of the 17 cases when it comes to the average of the total distance. In addition, the standard deviation of one-chromosome GA, TPGA, GA ${ }_{M L A}$, and EDA MLA $_{4}$ is $21187,33230,19785$, and 20041, respectively. It implies that the $\mathrm{EDA}_{\mathrm{MLA}}$ yields less variance than one-chromosome GA and TPGA. EDA ${ }_{M L A}$ might be more robust in terms of the average performance and the variance.

5.2. Maximum Traveling Distance Results. The maximum traveling distance was used as the second objective tested by the three algorithms. Thus, the algorithms minimized the loading of the salesman with the highest loading. As a result, this objective balanced the loading among the salesmen. As shown in Figure 4, both the $\mathrm{EDA}_{\mathrm{MLA}}$ and $\mathrm{GA}_{\mathrm{MLA}}$ remained superior to the one-chromosome GA and TPGA. A primary reason for these results could be the selection of a suitable salesman during the assignment phase according to the MLA rule. Hence, following this rule reduced the maximum traveling distance.

The assignment of 20 salesmen (see Figure 4) caused the lowest maximum loading on a salesman. This is a reasonable result because the loading is distributed over many salesmen. However, the assignment of 20 salesmen also resulted in the longest total traveling distance (see Section 5.1). Hence, the two objectives present a trade-off and should be considered simultaneously. In Figure 5, it shows the interaction plot between the method and the number of salesmen. This plot indicates $\mathrm{EDA}_{\mathrm{MLA}}$ and $\mathrm{GA}_{\mathrm{MLA}}$ perform well no matter how many salesmen are assigned. In addition, the number of salesmen yields the lower maximum traveling distance solved by the four algorithms.

Table 3 shows the complete results for the four algorithms. The $\mathrm{EDA}_{\mathrm{MLA}}$ and $\mathrm{GA}_{\mathrm{MLA}}$ are evidently superior to the one-chromosome GA and TPGA. The GA $A_{M L A}$ and $\mathrm{EDA}_{\mathrm{MLA}}$ have 20 and 14 lower mean values, respectively. This phenomenon indicates that the indirect encoding is better than the direct coding approach. The standard deviation of

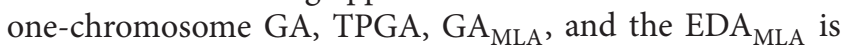
$14944,21728,13037$, and 12940, respectively. StDev indicates that the $\mathrm{EDA}_{\mathrm{MLA}}$ has less variation than $\mathrm{GA}_{\mathrm{MLA}}$ and TPGA. The $\mathrm{EDA}_{\mathrm{MLA}}$ might perform well in the minimization of the maximum traveling distance.

\section{Conclusions}

This study solves an in-group optimization problem that

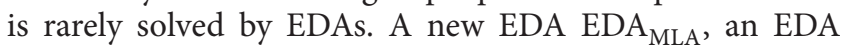
combined with the MLA rule, was proposed. Because the MLA rule was classified as transform-based encoding, the proposed algorithm was compared with the TPGA, the most favorable direct encoding strategy thus far. We evaluated these algorithms by solving the mTSP problem for 33 instances drawn from the TSPLIB. The scale of the experiments was larger than those of other mTSP studies. Our experimental results showed that the $\mathrm{EDA}_{\mathrm{MLA}}$ with the MLA rule outperformed the TPGA for both the objectives of total traveling and maximum traveling distance. Thus, the proposed algorithm is capable of efficiently solving the mTSP problem. In addition, the MLA rule was effective and could be applied with some GAs originally designed for 


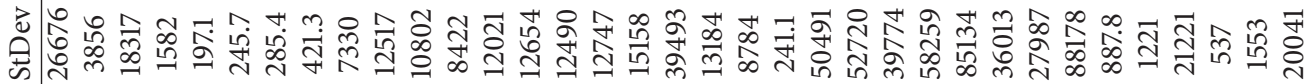

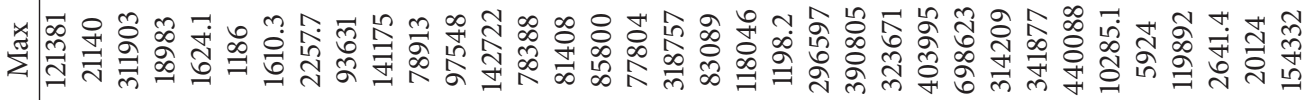

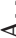

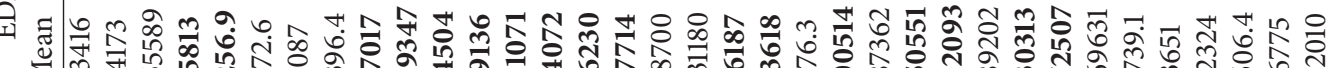

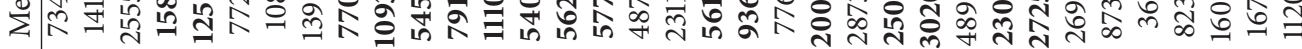

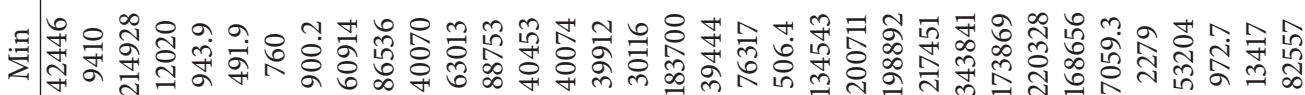

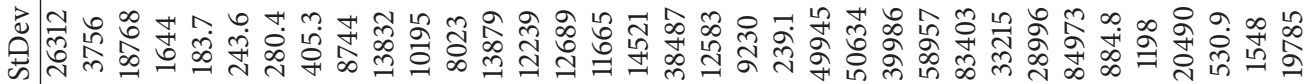

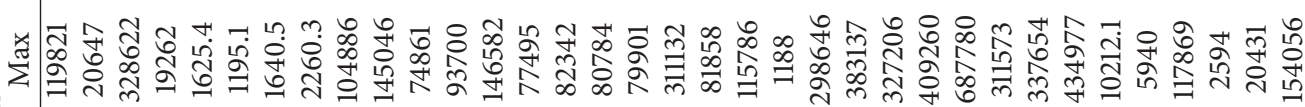
索

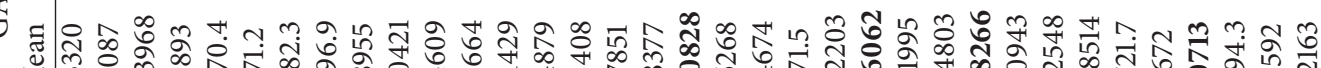

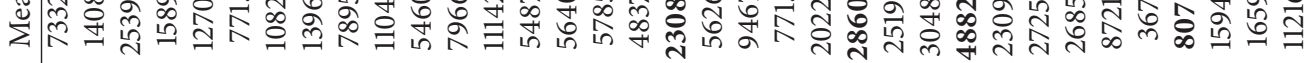

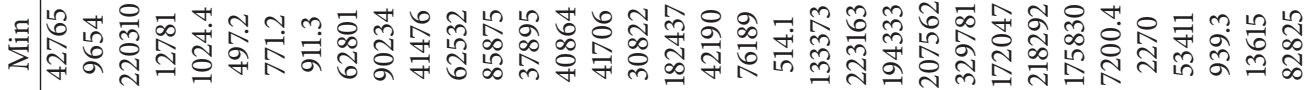

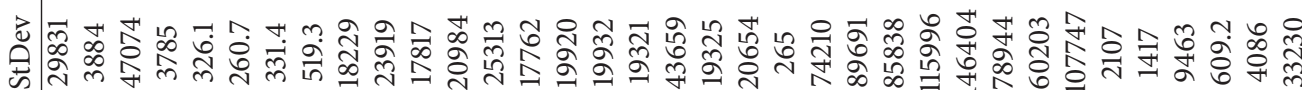

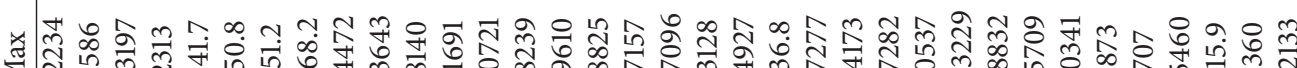
出

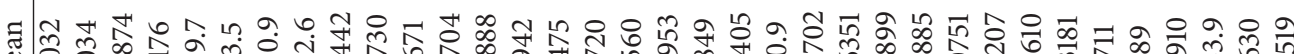

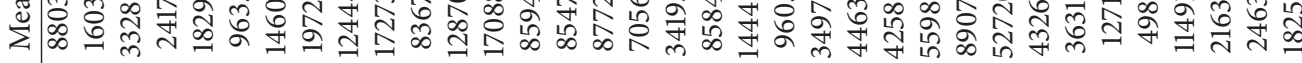

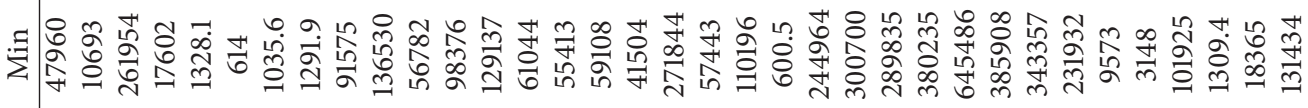

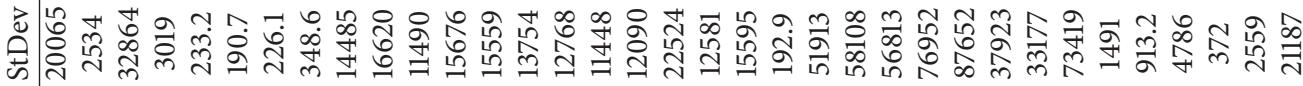

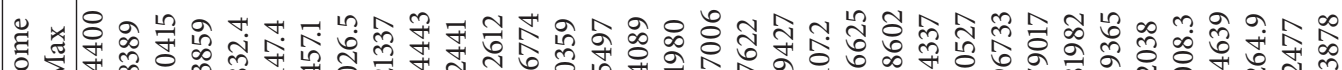

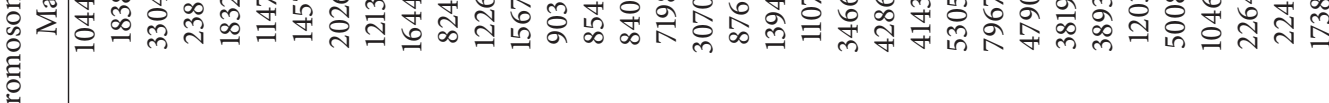

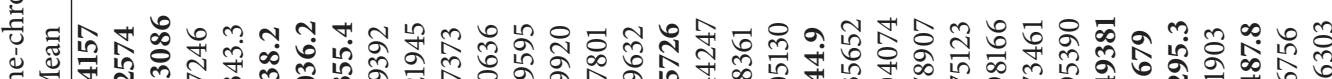

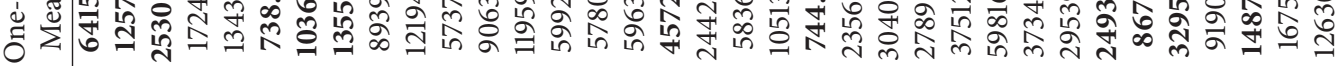

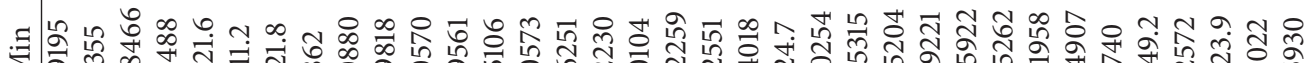

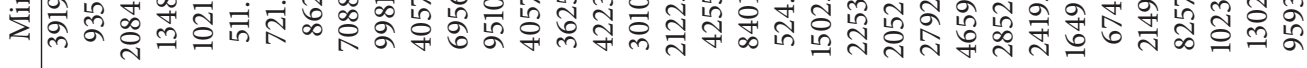

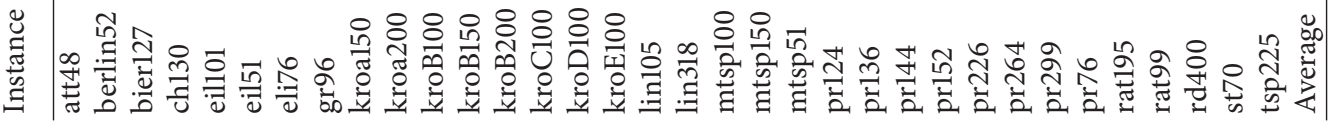




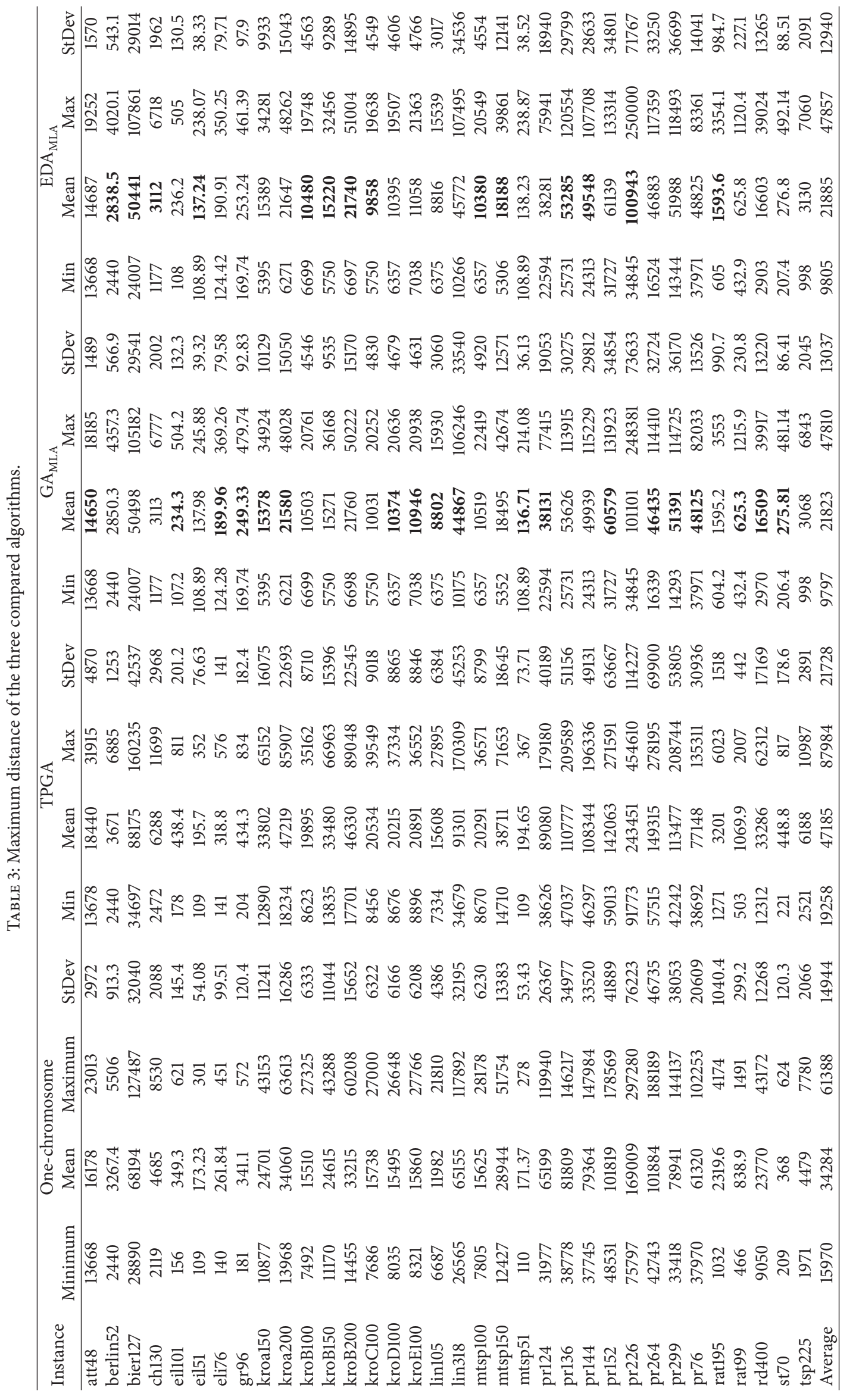




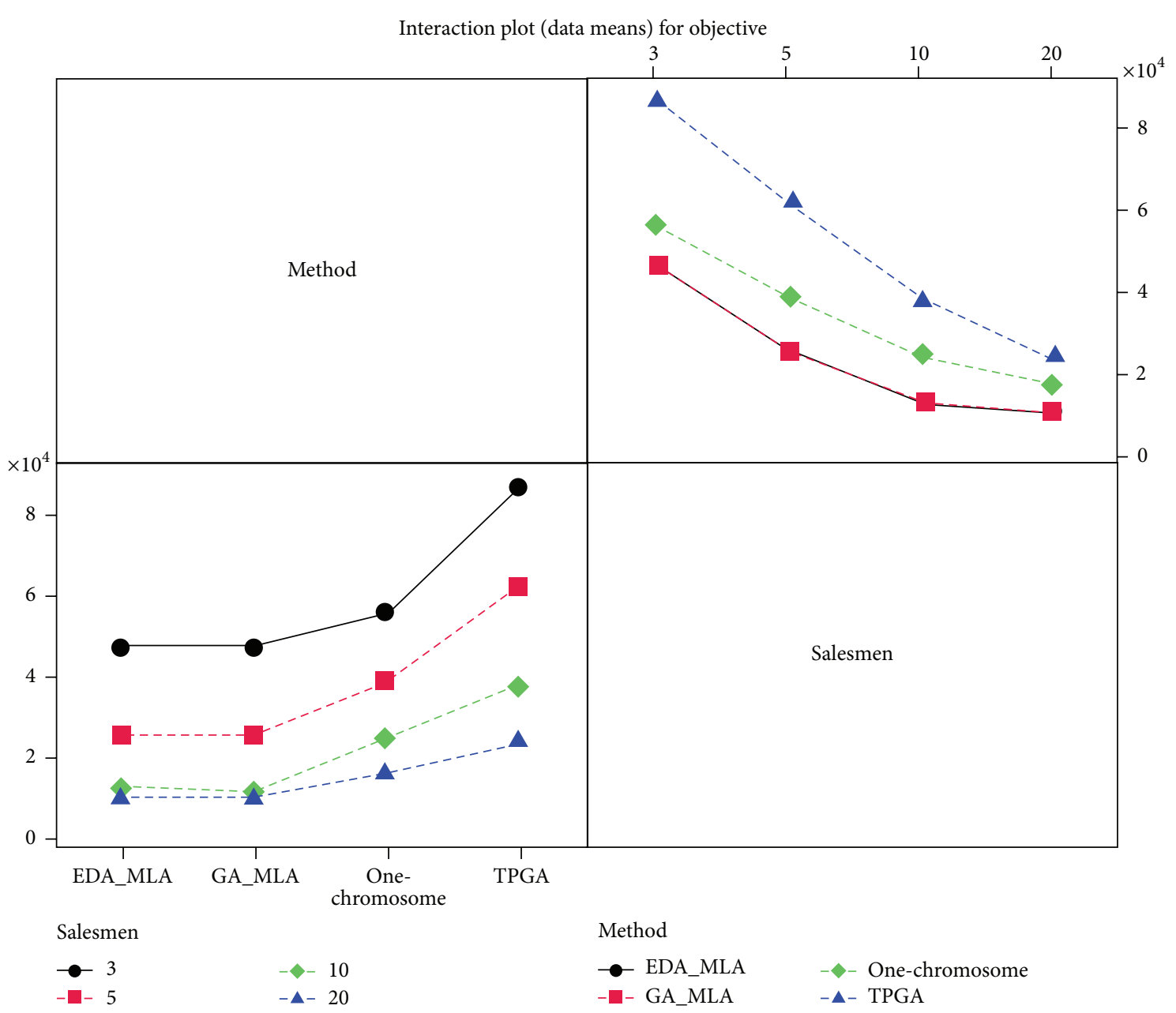

FIGURE 5: Interaction plot of the maximum distance for the compared algorithms.

permutation-type problems. As a result, this study provides insight for researchers investigating scheduling problems and advances the research on in-group optimization problems.

\section{Conflict of Interests}

The author declares that there is no conflict of interests regarding the publication of this paper.

\section{Acknowledgments}

The author would like to thank anonymous reviewers who enhance the quality of this paper and the Minister of Science and Technology with Grant nos. MOST 102-2221-E-230-019MY2 and MOST 101-2221-E-343-002.

\section{References}

[1] L. Ke, Q. Zhang, and R. Battiti, "MOEA/D-ACO: a multiobjective evolutionary algorithm using decomposition and AntColony," IEEE Transactions on Cybernetics, vol. 43, no. 6, pp. 1845-1859, 2013.
[2] J. Ceberio, E. Irurozki, A. Mendiburu, and J. A. Lozano, "A review on estimation of distribution algorithms in permutationbased combinatorial optimization problems," Progress in Artificial Intelligence, vol. 1, no. 1, pp. 103-117, 2012.

[3] M. Hauschild and M. Pelikan, "An introduction and survey of estimation of distribution algorithms," Swarm and Evolutionary Computation, vol. 1, no. 3, pp. 111-128, 2011.

[4] Q.-K. Pan and R. Ruiz, "An estimation of distribution algorithm for lot-streaming flow shop problems with setup times," Omega, vol. 40, no. 2, pp. 166-180, 2012.

[5] A. Zhou, F. Gao, and G. Zhang, "A decomposition based estimation of distribution algorithm for multiobjective traveling salesman problems," Computers \& Mathematics with Applications, vol. 66, no. 10, pp. 1857-1868, 2013.

[6] Q. Zhang, J. Sun, and E. Tsang, "An evolutionary algorithm with guided mutation for the maximum clique problem," IEEE Transactions on Evolutionary Computation, vol. 9, no. 2, pp. 192200, 2005.

[7] S.-H. Chen, M.-C. Chen, P.-C. Chang, Q. Zhang, and Y.M. Chen, "Guidelines for developing effective Estimation of Distribution Algorithms in solving single machine scheduling problems," Expert Systems with Applications, vol. 37, no. 9, pp. 6441-6451, 2010. 
[8] Y. Zhang and X. Li, "Estimation of distribution algorithm for permutation flow shops with total flowtime minimization," Computers \& Industrial Engineering, vol. 60, no. 4, pp. 706-718, 2011.

[9] S.-H. Chen and M.-C. Chen, "Addressing the advantages of using ensemble probabilistic models in Estimation of Distribution Algorithms for scheduling problems," International Journal of Production Economics, vol. 141, no. 1, pp. 24-33, 2013.

[10] V. A. Shim, K. C. Tan, and C. Y. Cheong, "A hybrid estimation of distribution algorithm with decomposition for solving the multiobjective multiple traveling salesman problem," IEEE Transactions on Systems, Man and Cybernetics C: Applications and Reviews, vol. 42, no. 5, pp. 682-691, 2012.

[11] T. Bektas, "The multiple traveling salesman problem: an overview of formulations and solution procedures," Omega, vol. 34, no. 3, pp. 209-219, 2006.

[12] S.-H. Chen, P.-C. Chang, T. C. E. Cheng, and Q. Zhang, "A selfguided genetic algorithm for permutation flowshop scheduling problems," Computers \& Operations Research, vol. 39, no. 7, pp. 1450-1457, 2012.

[13] A. E. Carter and C. T. Ragsdale, "A new approach to solving the multiple traveling salesperson problem using genetic algorithms," European Journal of Operational Research, vol. 175, no. 1, pp. 246-257, 2006.

[14] B. Gavish and K. Srikanth, "An optimal solution method for large-scale multiple traveling salesmen problems," Operations Research, vol. 34, no. 5, pp. 698-717, 1986.

[15] G. Laporte and Y. Nobert, "A cutting planes algorithm for the $m$ salesmen problem," Operational Research Society Journal, vol. 31, no. 11, pp. 1017-1023, 1980.

[16] D. L. Miller and J. F. Pekny, "Exact solution of large asymmetric traveling salesman problems," Science, vol. 251, no. 4995, pp. 754-761, 1991.

[17] L. Tang, J. Liu, A. Rong, and Z. Yang, "A multiple traveling salesman problem model for hot rolling scheduling in Shanghai Baoshan Iron \& Steel Complex," European Journal of Operational Research, vol. 124, no. 2, pp. 267-282, 2000.

[18] C. J. Malmborg, "A genetic algorithm for service level based vehicle scheduling," European Journal of Operational Research, vol. 93, no. 1, pp. 121-134, 1996.

[19] Y.-B. Park, "A hybrid genetic algorithm for the vehicle scheduling problem with due times and time deadlines," International Journal of Production Economics, vol. 73, no. 2, pp. 175-188, 2001.

[20] E. C. Brown, C. T. Ragsdale, and A. E. Carter, "A grouping genetic algorithm for the multiple traveling salesperson problem," International Journal of Information Technology and Decision Making, vol. 6, no. 2, pp. 333-347, 2007.

[21] A. Király and J. Abonyi, "A novel approach to solve multiple traveling sales-men problem by genetic algorithm," in Computational Intelligence in Engineering, pp. 141-151, Springer, 2010.

[22] A. Singh and A. S. Baghel, "A new grouping genetic algorithm approach to the multiple traveling salesperson problem," Soft Computing, vol. 13, no. 1, pp. 95-101, 2009.

[23] J.-P. Arnaout, G. Rabadi, and R. Musa, "A two-stage ant colony optimization algorithm to minimize the makespan on unrelated parallel machines with sequence-dependent setup times," Journal of Intelligent Manufacturing, vol. 21, no. 6, pp. 693-701, 2010.

[24] S.-H. Chen and M.-C. Chen, "Operators of the two-part encoding genetic algorithm in solving the multiple traveling salesmen problem," in Proceedings of the 16th Annual Conference on
Technologies and Applications of Artificial Intelligence (TAAI '11), pp. 331-336, November 2011.

[25] E. Vallada and R. Ruiz, "A genetic algorithm for the unrelated parallel machine scheduling problem with sequence dependent setup times," European Journal of Operational Research, vol. 211, no. 3, pp. 612-622, 2011.

[26] A. H. Kashan, M. H. Kashan, and S. Karimiyan, "A particle swarm optimizer for grouping problems," Information Sciences, vol. 252, pp. 81-95, 2013.

[27] T. W. Liao, P. C. Chang, R. J. Kuo, and C.-J. Liao, "A comparison of five hybrid metaheuristic algorithms for unrelated parallelmachine scheduling and inbound trucks sequencing in multidoor cross docking systems," Applied Soft Computing, vol. 21, pp. 180-193, 2014.

[28] R. Ruiz and C. Maroto, "A genetic algorithm for hybrid flowshops with sequence dependent setup times and machine eligibility," European Journal of Operational Research, vol. 169, no. 3, pp. 781-800, 2006.

[29] P.-C. Chang and S.-H. Chen, "Integrating dominance properties with genetic algorithms for parallel machine scheduling problems with setup times," Applied Soft Computing, vol. 11, no. 1, pp. 1263-1274, 2011.

[30] S.-Y. Wang, L. Wang, M. Liu, and Y. Xu, "An effective estimation of distribution algorithm for solving the distributed permutation flow-shop scheduling problem," International Journal of Production Economics, vol. 145, no. 1, pp. 387-396, 2013.

[31] A. Salhi, J. A. V. Rodríguez, and Q. Zhang, "An estimation of distribution algorithm with guided mutation for a complex flow shop scheduling problem," in Proceedings of the 9th Annual Genetic and Evolutionary Computation Conference (GECCO '07), pp. 570-576, ACM, July 2007.

[32] M. Pelikan, D. E. Goldberg, and F. G. Lobo, "A survey of optimization by building and using probabilistic models," Computational Optimization and Applications, vol. 21, no. 1, pp. 5-20, 2002.

[33] J. A. Lozano, P. Larranaga, and I. Inza, Towards a New Evolutionary Computation: Advances in the Estimation of Distribution Algorithms, Springer, Berlin, Germany, 2006.

[34] P.-C. Chang, S.-S. Chen, and C.-Y. Fan, "Mining gene structures to inject artificial chromosomes for genetic algorithm in single machine scheduling problems," Applied Soft Computing Journal, vol. 8, no. 1, pp. 767-777, 2008.

[35] J. L. Shapiro, "Diversity loss in general estimation of distribution algorithms," in Parallel Problem Solving from Nature-PPSN IX, vol. 4193 of Lecture Notes in Computer Science, pp. 92-101, Springer, Berlin, Germany, 2006.

[36] J. Branke, C. Lode, and J. L. Shapiro, "Addressing sampling errors and diversity loss in UMDA," in Proceedings of the 9th Annual Genetic and Evolutionary Computation Conference (GECCO '07), pp. 508-515, ACM, July 2007.

[37] B. Jarboui, M. Eddaly, and P. Siarry, "An estimation of distribution algorithm for minimizing the total flowtime in permutation flowshop scheduling problems," Computers and Operations Research, vol. 36, no. 9, pp. 2638-2646, 2009.

[38] P. Hansen and N. Mladenovi, "Variable neighborhood search: principles and applications," European Journal of Operational Research, vol. 130, no. 3, pp. 449-467, 2001.

[39] S. Chen, P. Chang, Q. Zhang, and C. Wang, "A guided memetic algorithm with probabilistic models," International Journal of Innovative Computing, Information and Control, vol. 5, no. 12, pp. 4753-4764, 2009. 
[40] S. Chen, P. C. Chang, M. C. Chen, and Y. M. Chen, "A selfguided genetic algorithm with dominance properties for single machine scheduling problems," in Proceedings of the IEEE Symposium on Computational Intelligence in Scheduling (CISched '09), pp. 76-83, IEEE, Nashville, Tenn, USA, April 2009.

[41] S.-H. Chen, P.-C. Chang, T. C. E. Cheng, and Q. Zhang, "A selfguided genetic algorithm for permutation flowshop scheduling problems," Computers and Operations Research, vol. 39, no. 7, pp. 1450-1457, 2012.

[42] I. Good, The Estimation of Probabilities: An Essay on Modern Bayesian Methods, The MIT Press, 2003.

[43] J. Han and M. Kamber, Data Mining: Concepts and Techniques, Morgan Kaufmann, 2006.

[44] B. Cestnik, "Estimating probabilities: a crucial task in machine learning," in Proceedings of the 9th European Conference on Artificial Intelligence, pp. 147-149, 1990.

[45] S. Baluja and S. Davies, "Fast probabilistic modeling for combinatorial optimization," in Proceedings of the 15th National Conference on Artificial Intelligence, pp. 469-476, John Wiley \& Sons Inc, July 1998.

[46] Y. Chan and S. F. Baker, "The multiple depot, multiple traveling salesmen facility-location problem: vehicle range, service frequency, and heuristic implementations," Mathematical and Computer Modelling, vol. 41, no. 8-9, pp. 1035-1053, 2005.

[47] S. Ghafurian and N. Javadian, "An ant colony algorithm for solving fixed destination multi-depot multiple traveling salesmen problems," Applied Soft Computing Journal, vol. 11, no. 1, pp. 1256-1262, 2011.

[48] S. Yadlapalli, W. A. Malik, S. Darbha, and M. Pachter, "A Lagrangian-based algorithm for a multiple depot, multiple traveling salesmen problem," Nonlinear Analysis: Real World Applications, vol. 10, no. 4, pp. 1990-1999, 2009.

[49] S. Gorenstein, "Printing press scheduling for multi-edition periodicals," Management Science, vol. 16, no. 6, pp. 373-383, 1970.

[50] A. E. Carter and C. T. Ragsdale, "Scheduling pre-printed newspaper advertising inserts using genetic algorithms," Omega, vol. 30, no. 6, pp. 415-421, 2002.

[51] J. A. Svestka and V. E. Huckfeldt, "Computational experience with an m-salesman traveling salesman algorithm," Management Science, vol. 19, no. 7, pp. 790-799, 1973.

[52] J. K. Lenstra and A. H. G. R. Kan, "Some simple applications of the travelling salesman problem," Operational Research Quarterly, vol. 26, no. 4, pp. 717-733, 1975.

[53] T. Zhang, W. A. Gruver, and M. H. Smith, "Team scheduling by genetic search," in Proceedings of the 2nd International Conference on Intelligent Processing and Manufacturing of Materials (IPMM '99), vol. 2, pp. 839-844, Honolulu, Hawaii, USA, 1999.

[54] K. C. Gilbert and R. B. Hofstra, "A new multiperiod multiple traveling salesman problem with heuristic and application to a scheduling problem," Decision Sciences, vol. 23, no. 1, pp. 250259, 1992.

[55] C. Okonjo-Adigwe, "An effective method of balancing the workload amongst salesmen," Omega, vol. 16, no. 2, pp. 159-163, 1988.

[56] R. Wolfler Calvo and R. Cordone, "A heuristic approach to the overnight security service problem," Computers \& Operations Research, vol. 30, no. 9, pp. 1269-1287, 2003.

[57] R. D. Angel, W. L. Caudle, R. Noonan, and A. Whinston, "Computer-assisted school bus scheduling," Management Science, vol. 18, no. 6, pp. 279-288, 1972.
[58] K. H. Kim and Y.-M. Park, "A crane scheduling method for port container terminals," European Journal of Operational Research, vol. 156, no. 3, pp. 752-768, 2004.

[59] X. Wang and A. C. Regan, "Local truckload pickup and delivery with hard time window constraints," Transportation Research Part B: Methodological, vol. 36, no. 2, pp. 97-112, 2002.

[60] X. Gan, Y. Wang, S. Li, and B. Niu, "Vehicle routing problem with time windows and simultaneous delivery and pick-up service based on MCPSO," Mathematical Problems in Engineering, vol. 2012, Article ID 104279, 11 pages, 2012.

[61] X. Gan, Y. Wang, J. Kuang, Y. Yu, and B. Niu, "Emergency vehicle scheduling problem with time utility in disasters," Mathematical Problems in Engineering, vol. 2015, Article ID 164194, 7 pages, 2015.

[62] B. Brumitt and A. Stentz, "Dynamic mission planning for multiple mobile robots," Intelligent Unmanned Ground Vehicles, vol. 388, pp. 221-234, 1997.

[63] B. L. Brumitt and A. Stentz, "GRAMMPS: a generalized mission planner for multiple mobile robots in unstructured environments," in Proceedings of the IEEE International Conference on Robotics and Automation, vol. 2, pp. 1564-1571, IEEE, May 1998.

[64] Z. Yu, L. Jinhai, G. Guochang, Z. Rubo, and Y. Haiyan, "An implementation of evolutionary computation for path planning of cooperative mobile robots," in Proceedings of the 4th World Congress on Intelligent Control and Automation, vol. 3, pp. 1798 1802, IEEE, 2002.

[65] A. Basu, A. Elnagar, and R. Al-Hajj, "Efficient coordinated motion," Mathematical and Computer Modelling, vol. 31, no. 2-3, pp. 39-53, 2000.

[66] J. L. Ryan, T. G. Bailey, J. T. Moore, and W. B. Carlton, "Reactive Tabu Search in unmanned aerial reconnaissance simulations," in Proceedings of the 30th Conference on Winter Simulation, pp. 873-880, IEEE Computer Society Press, December 1998.

[67] H. A. Saleh and R. Chelouah, "The design of the global navigation satellite system surveying networks using genetic algorithms," Engineering Applications of Artificial Intelligence, vol. 17, no. 1, pp. 111-122, 2004. 


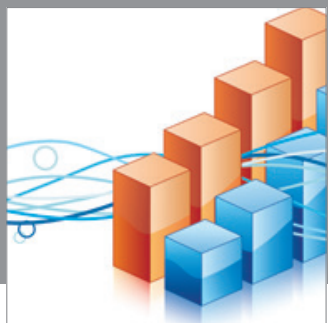

Advances in

Operations Research

mansans

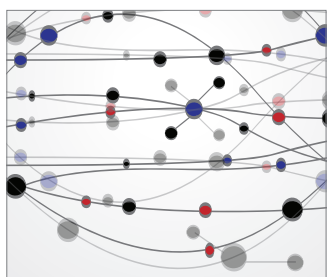

The Scientific World Journal
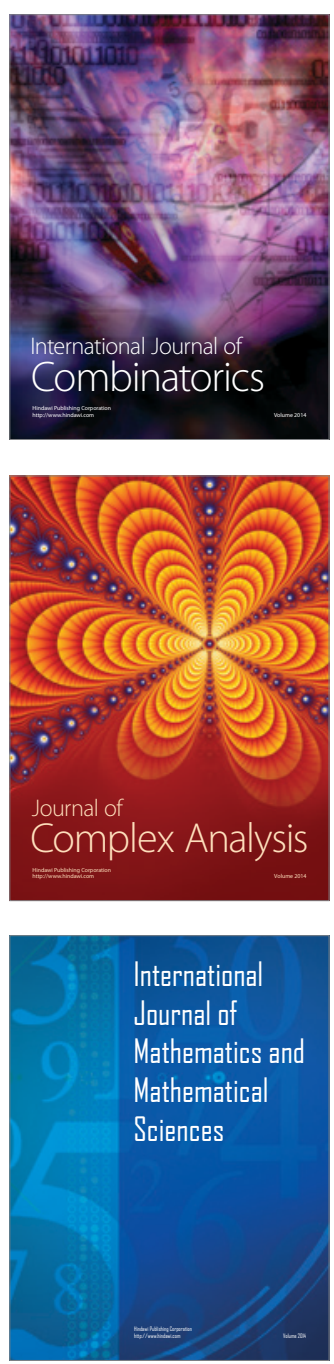
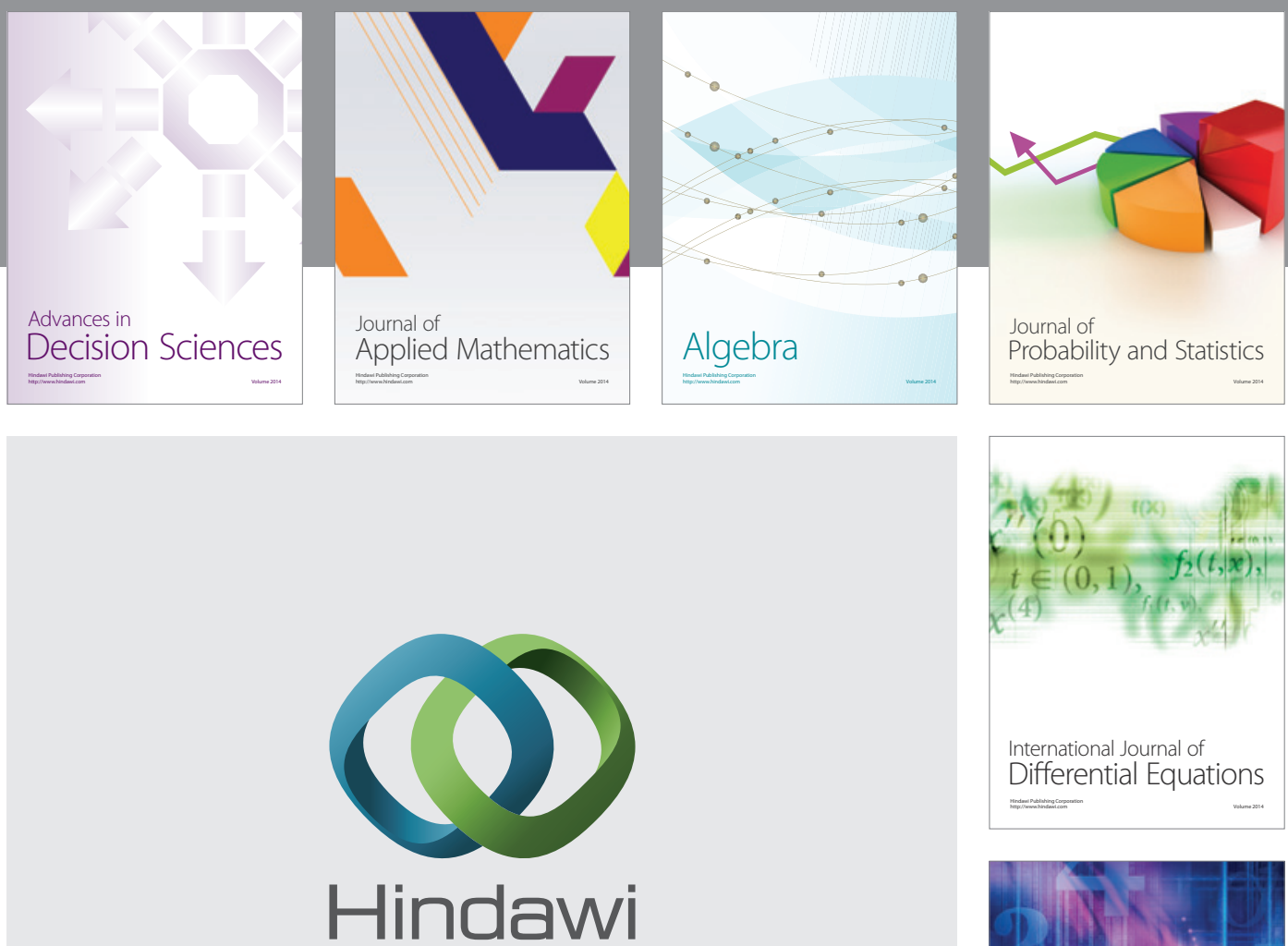

Submit your manuscripts at http://www.hindawi.com
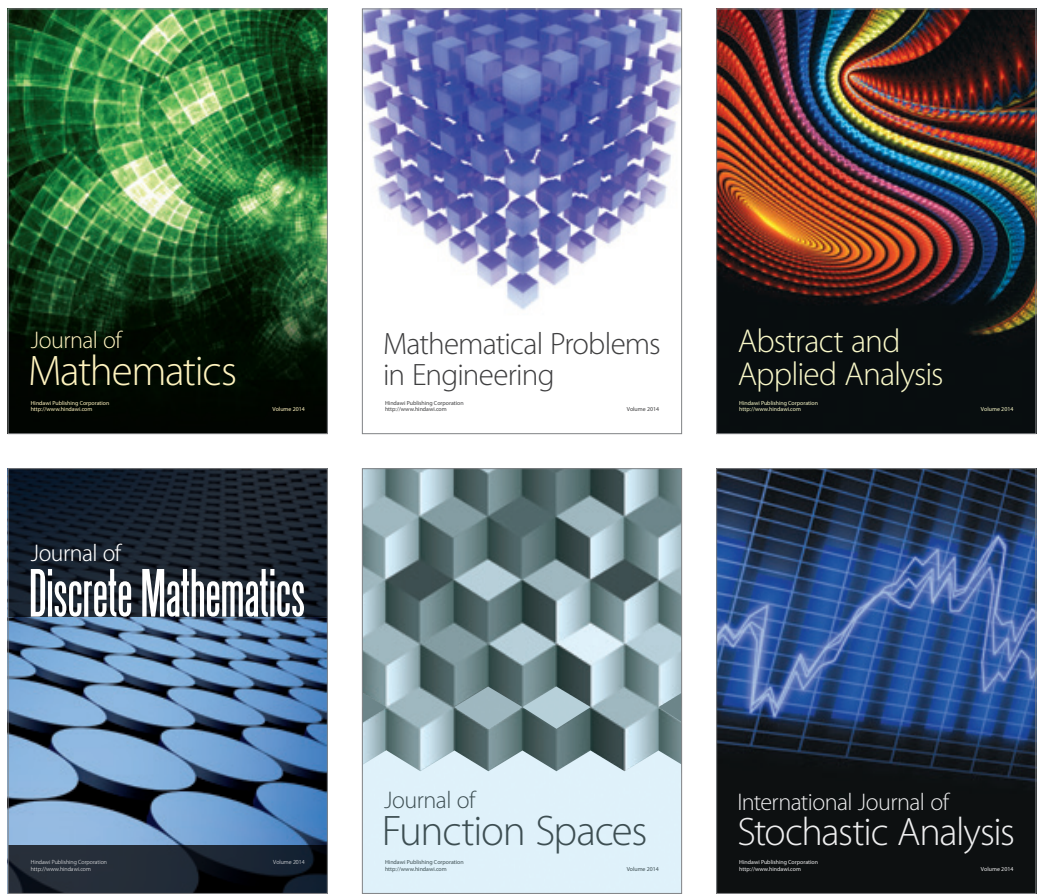

Journal of

Function Spaces

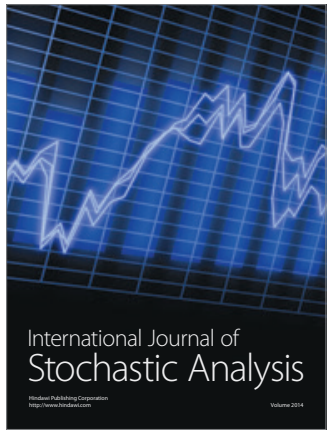

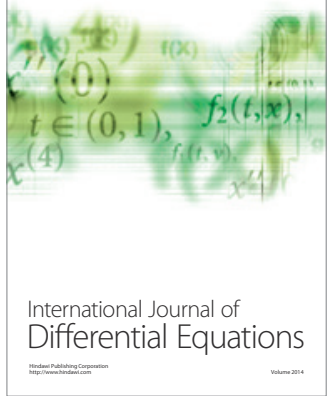
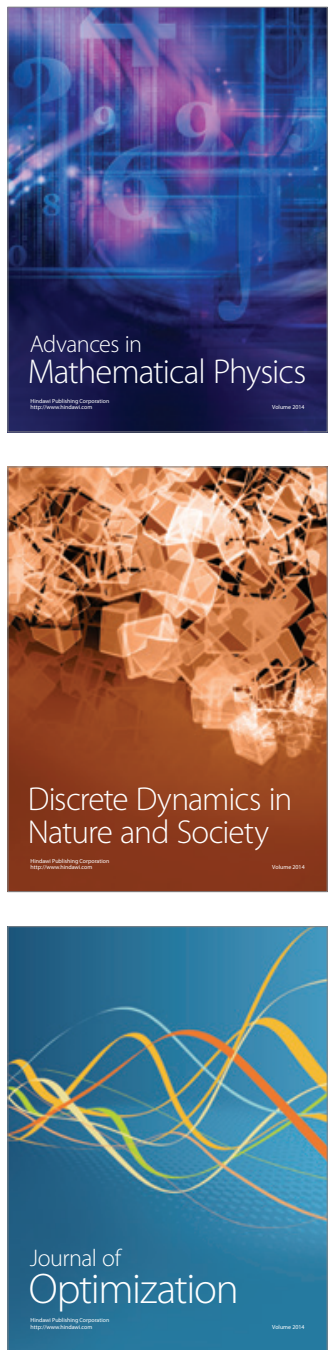